\title{
Реле от компании Fanhar: высокое качество по конкурентным ценам
}

\author{
Ф. Комиссаров ${ }^{1}$
}

УДК 621.318.5 | ВАК 05.27.01

Высокотехнологичная компания Fanhar Electronics, расположенная в китайской провинции Чжэцзян (г. Цзясин), занимается разработкой и производством электрических реле. В настоящее время в ассортимент выпускаемой продукции входит более 30 видов реле сотен типоразмеров, предназначенных для применения в таких областях, как телекоммуникации, умный дом, промышленные системы управления, бытовая электроника, промышленная автоматизация и фотоэлектрические источники энергии. Изделия компании поставляются не только на внутренний рынок, но и в Южную Корею, Израиль, США, Аргентину, Великобританию, Россию, Индию и другие страны. Благодаря совершенной системе обеспечения качества, тщательному преди постпродажному обслуживанию, а также обширной сервисной сети компания зарекомендовала себя надежным поставщиком продукции с оптимальным соотношением цены и качества.

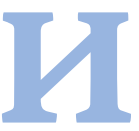
меющая 20-летний опыт разработки и производства реле компания Fanhar располагает восемью полностью автоматизированными линиями, производительность которых достигает 80 млн штук в год (рис. 1). Короткие сроки изготовления и поддержка постоянного складского запаса готовой продукции позволяют обеспечить бесперебойность поставок.

Постоянный контроль технологическихпроцессов гарантирует стабильно высокое качество продукции. С помощью современного тестового оборудования на предприятии можно проводить широкий спектр испытаний готовой продукции, в частности испытания на ускоренное старение под воздействием температуры, на стойкость при повышенной влажности в условиях высокой температуры, на долговечность, воспламеняемость, климатические испытания (тепло, холод), а также измерения сопротивления, чувствительности и нагрузочной способности реле.

Компания имеет сертификаты систем менеджмента качества ISO 9001, ISO 14001, ее продукция удовлетворяет требованиям стандарта REACH, директиве RoHS
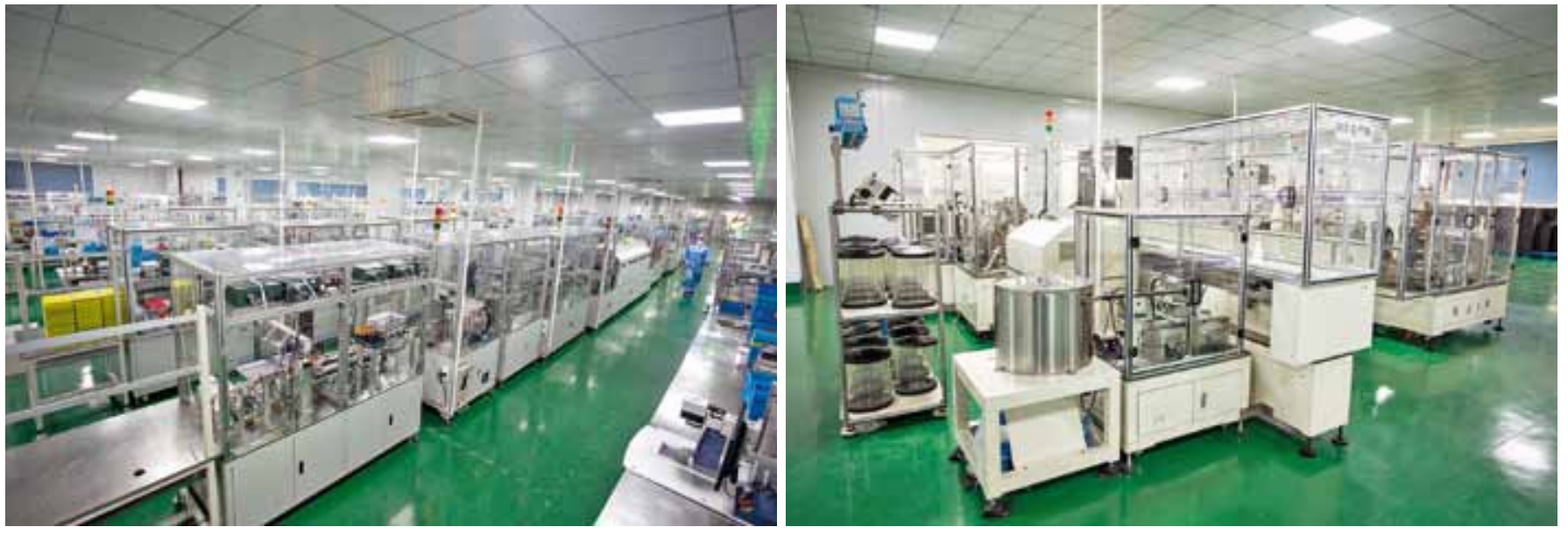

Рис. 1. Производственные участки компании Fanhar 
и сертифицирована по UL, TUV и CQC. Fanhar запатентовала более 30 типов реле, большая часть которых по техническим характеристикам не уступает изделиям таких мировых производителей, как Hongfa, Omron, Tyco/Shrack, Fujitsu, Panasonic.
В каталоге продукции Fanhar представлено более 40 типов реле (табл. 1), что позволяет использовать их в качестве замены аналогичных или полностью совместимых реле более дорогостоящих брендов. Большую часть типов реле из продуктовой линейки можно

Таблица 1. Реле компании Fanhar и их аналоги

\begin{tabular}{|c|c|c|c|c|c|}
\hline \multirow{2}{*}{$\begin{array}{l}\text { Модель } \\
\text { Fanhar }\end{array}$} & \multirow[t]{2}{*}{ Номинальная нагрузка } & \multirow{2}{*}{$\begin{array}{l}\text { Тип } \\
\text { контактов }\end{array}$} & \multicolumn{3}{|c|}{ Аналогичная модель } \\
\hline & & & Hongfa & Omron & Тусо \\
\hline W11 & $5 \mathrm{~A} / 250 \mathrm{~B} \mathrm{AC}, 10 \mathrm{~A} / 250 \mathrm{~B} \mathrm{AC}$ & $1 \mathrm{~A}, 1 \mathrm{C}$ & $\mathrm{HF} 32 \mathrm{~F} / 32 \mathrm{FA}$ & G6B & OJ/OJE \\
\hline W12 & 30 A / 250 B AC, 20 A/ 30 В DC (тип BС) & $1 \mathrm{~A}, 1 \mathrm{C}$ & HF105-1/165FD & G8P & Т9А / Т90 \\
\hline W12T & 40 A/ 250 B AC, 20 A/ 30 В DC (тип BG) & $1 \mathrm{~A}$ & HF165F & - & T9S \\
\hline W12TP & $30 \mathrm{~A} / 250 \mathrm{~B} \mathrm{AC}, 20 \mathrm{~A} / 30 \mathrm{~B} \mathrm{DC}$ & $1 \mathrm{~A}, 1 \mathrm{~B}, 1 \mathrm{C}$ & HF105-5 & G8P/G7G & Т9А / Т90 \\
\hline W14 & $10 \mathrm{~A} / 250 \mathrm{~B} \mathrm{AC}, 15 \mathrm{~A} / 125 \mathrm{~B} \mathrm{AC}$ & $1 \mathrm{~A}, 1 \mathrm{C}$ & $\mathrm{HF} 3 \mathrm{FA} / \mathrm{FD} / \mathrm{FF}$ & G5LA & $\mathrm{PCE} / \mathrm{T} 7 \mathrm{~S}$ \\
\hline W14T & $15 \mathrm{~A} / 250 \mathrm{~B} \mathrm{AC}, 20 \mathrm{~A} / 125 \mathrm{~B} \mathrm{AC}$ & $1 \mathrm{~A}, 1 \mathrm{C}$ & HF21FF & G5L & SRUDH \\
\hline W15-1 & $16 \mathrm{~A} / 250 \mathrm{~B} \mathrm{AC}, 12 \mathrm{~A} / 250 \mathrm{~B} \mathrm{AC}$ & $1 \mathrm{~A}, 1 \mathrm{~B}, 1 \mathrm{C}$ & HF115F & G2RL & $\mathrm{RT}$ \\
\hline W15-2 & $8 \mathrm{~A} / 250 \mathrm{~B} \mathrm{AC}, 10 \mathrm{~A} / 125 \mathrm{~B} \mathrm{AC}$ & $2 \mathrm{~A}, 2 \mathrm{~B}, 2 \mathrm{C}$ & HF115F & G2RL & $\mathrm{RT}$ \\
\hline W15L & $16 \mathrm{~A} / 250 \mathrm{~B} \mathrm{AC}, 20 \mathrm{~A} / 250 \mathrm{~B} \mathrm{AC}$ & $1 \mathrm{~A}, 1 \mathrm{~B}, 1 \mathrm{C}$ & HFE20 & G5RL & $\mathrm{RT}$ \\
\hline W16 & $5 \mathrm{~A} / 250 \mathrm{~B} \mathrm{AC}, 10 \mathrm{~A} / 250 \mathrm{~B} \mathrm{AC}$ & $1 \mathrm{~A}, 1 \mathrm{C}$ & HF33 & G5Q & $\mathrm{PCH}$ \\
\hline W18 & $5 \mathrm{~A} / 250 \mathrm{~B} \mathrm{AC}$ & $1 \mathrm{~A}$ & HF46F & G5NB & PCJ \\
\hline W19/W19F & $3 \mathrm{~A} / 250 \mathrm{~B} \mathrm{AC}, 5 \mathrm{~A} / 250 \mathrm{~B} \mathrm{AC}$ & $1 \mathrm{~A}$ & HF49FD & G6DS & $\mathrm{PCH}$ \\
\hline W20 & $20 \mathrm{~A} / 250 \mathrm{~B} \mathrm{AC}, 25 \mathrm{~A} / 250 \mathrm{~B} \mathrm{AC}$ & $1 \mathrm{~A}$ & HF161F & $\mathrm{G} 4 \mathrm{~A}$ & PCFN \\
\hline W21L & $90 \mathrm{~A} / 250 \mathrm{~B} \mathrm{AC}$ & $1 \mathrm{~B}$ & HFE19-90 & - & - \\
\hline W22L & $10 \mathrm{~A} / 250 \mathrm{~B} \mathrm{AC}, 16 \mathrm{~A} / 250 \mathrm{~B} \mathrm{AC}$ & $1 \mathrm{~A}, 1 \mathrm{~B}, 1 \mathrm{C}$ & $508 \mathrm{E}$ & - & - \\
\hline W23L & $60 \mathrm{~A} / 250 \mathrm{~B} \mathrm{AC}$ & $1 \mathrm{~A}, 1 \mathrm{~B}$ & HFE19-60 & - & - \\
\hline W26L & $40 \mathrm{~A} / 25 \mathrm{~A} / 250 \mathrm{~B} \mathrm{AC}$ & $1 \mathrm{~A}, 1 \mathrm{~B}, 1 \mathrm{C}$ & HFE26 & - & - \\
\hline W27L & $100 \mathrm{~A} / 250 \mathrm{~B} \mathrm{AC}, 120 \mathrm{~A} / 250 \mathrm{~B} \mathrm{AC}$ & $1 \mathrm{~A}, 1 \mathrm{~B}$ & HFE29 & - & - \\
\hline W28 & $20 \mathrm{~A} / 14 \mathrm{~B} \mathrm{DC}, 5 \mathrm{~A} / 250 \mathrm{~B} \mathrm{AC}$ & $1 \mathrm{~A}, 1 \mathrm{C}$ & HFKW & - & - \\
\hline W29LA & $100 \mathrm{~A} / 250 \mathrm{~B} \mathrm{AC}$ & $3 \mathrm{~A}, 3 \mathrm{~B}$ & HFE23 & - & - \\
\hline W30L & $40 \mathrm{~A} / 250 \mathrm{~B} \mathrm{AC}, 50 \mathrm{~A} / 250 \mathrm{~B} \mathrm{AC}$ & $1 \mathrm{~A}, 1 \mathrm{~B}, 1 \mathrm{C}$ & HFE10 & - & - \\
\hline W31L & $100 \mathrm{~A} / 250 \mathrm{~B} \mathrm{AC}$ & 2B & HFE28 & - & - \\
\hline W32 & $1 \mathrm{~A} / 125 \mathrm{~B} \mathrm{AC}, 2 \mathrm{~A} / 30 \mathrm{~B} \mathrm{DC}$ & $2 \mathrm{C}$ & HED27 & G5V-2 & - \\
\hline W33 & $5 \mathrm{~A} / 250 \mathrm{~B} \mathrm{AC}$ & $1 \mathrm{~A}$ & HF36FD & G5A-1 & SDT \\
\hline W35L & $16 \mathrm{~A} / 250 \mathrm{~B} \mathrm{AC}$ & $2 \mathrm{~A}, 2 \mathrm{~B}$ & HFE39 & - & - \\
\hline W36 & $10 \mathrm{~A} / 250 \mathrm{~B} \mathrm{AC}, 16 \mathrm{~A} / 250 \mathrm{~B} \mathrm{AC}$ & $1 \mathrm{~A}$ & HF7520 & G5CA & PCD \\
\hline W37-1 & $15 \mathrm{~A} / 250 \mathrm{~B} \mathrm{AC}$ & $1 \mathrm{~A}, 1 \mathrm{C}$ & HF13F & - & - \\
\hline W37-2 & $10 \mathrm{~A} / 250 \mathrm{~B} \mathrm{AC}$ & $2 \mathrm{~A}, 2 \mathrm{C}$ & HF13F & - & - \\
\hline W38/38A & $1 \mathrm{~A} / 250 \mathrm{~B} \mathrm{AC}, 3 \mathrm{~A} / 125 \mathrm{~B} \mathrm{AC}$ & $1 C$ & HFD41 / 41A & G2E & OUA / OUAZ \\
\hline
\end{tabular}



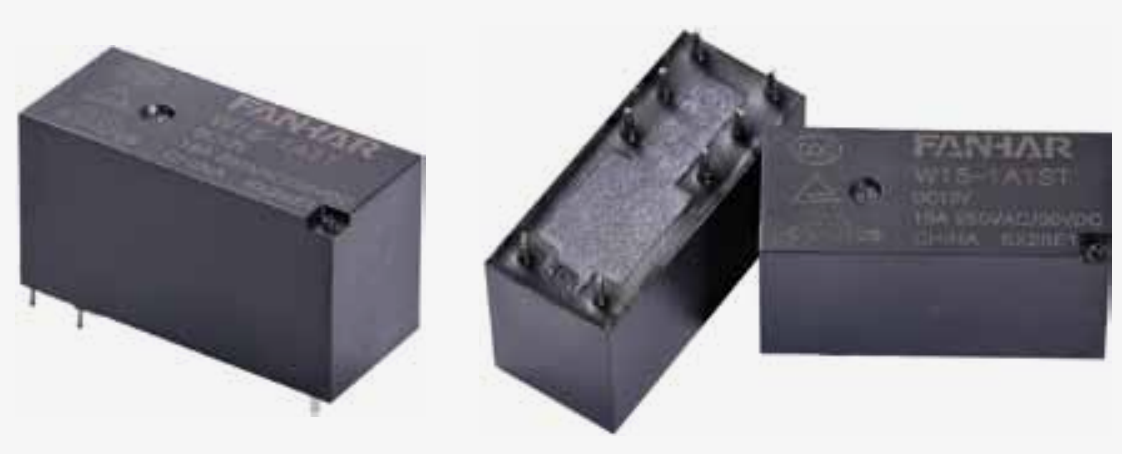

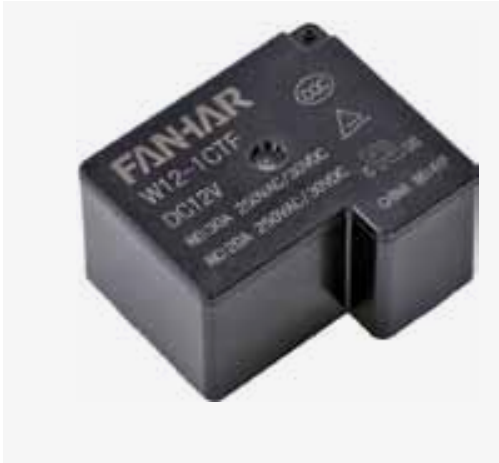

Рис. 4. Силовое реле серии W12
Рис. 2. Силовые реле серии W15-1

усовершенствовать по индивидуальным требованиям заказчика.

Наиболее популярные в продуктовой линейке Fanhar силовые реле серий W15-1/W15-2 (рис. 2), которые являются полными аналогами серий HF115F (Hongfa), G2RL (Omron), RT (Tyco/Shrack), FTRK1/FTRFl (Fujitsu), LZ (Panasonic). Kpoме того, в ассортименте продукции представлены поляризованные (latching) реле W15L (рис. 3), предназначенныедля автоматизированных систем коммерческого учета электроэнергии (АСКУЭ) и электросчетчиков. В системах промышленного управления и фотоэлектрических системах востребованы также силовые реле серии W12 (рис. 4). По требованию заказчика устройства могут быть выполнены в герметичных пластиковых корпусах, которые подходят для применения в различных климатических условиях.

Продукция Fanhar разработана с учетом последних достижений в области конструирования реле и с применением новейших материалов. Контакты реле выполнены из экологичных материалов повышенной твердости с использованием износостойкого антиплавкого соединения AgSnOInO с контактной нагрузкой более 5 A. Устройства могут коммутировать напряжение более 440 В АС и 300 B DC, а также ток более 120 A.

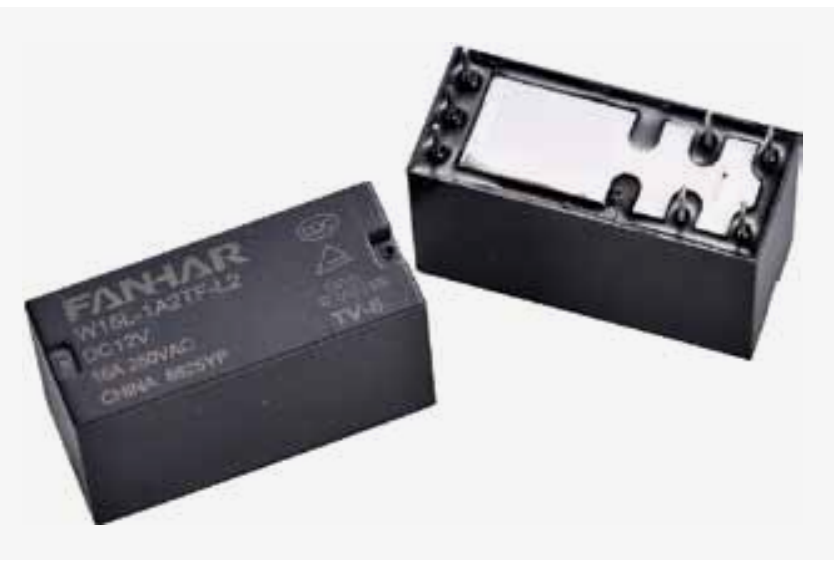

Рис. 3. Поляризованное реле серии W15L
Реле оснащены чувствительными катушками, мощность обмоток которых превышает 0,12 Вт. Диапазон рабочих температур большей части изделий $-40 . .105^{\circ} \mathrm{C}$. Ресурс срабатываний реле превышает 50 тыс. для катушек переменного тока и 100 тыс. - для катушек постоянного тока.

Высокие характеристики реле обеспечиваются благодаря применению передовых технологических и конструкторских решений с учетом накопленного в отрасли опыта.

В качестве материала неподвижной контактной пружины Т2 используется чистая медь, которая отличается высокой электропроводностью (удельная проводимость меди составляет 97\% IACS, что обеспечивает малый нагрев изделия). Подвижная контактная пружина изготавливается из сплава мышьяка и меди (Mitsubishi MSPl) (рис. 5). При высокой температуре этот материал сохраняет упругую устойчивость, его удельная проводимость составляет 55\% IACS, что обеспечивает стабильную работу при длительной подаче напряжения.

Для изготовления пластиковых термостойких деталей стандартных размеров (держателя катушки, корпуса) применяется материал Mitsubishi PBT5010GN6-30M8, который устойчив к температурам около $200^{\circ} \mathrm{C}$; для пластин и основания - материал LCP6807LHE с термостойкостью порядка $260^{\circ} \mathrm{C}$.

Пластиковые детали стандартных размеров изготавливаются обычно по технологии литья под давлением гидравлического типа, которая не всегда отвечает требованиям по точности обработки. Поэтому в компании Fanhar применяют литье электродинамического типа, чтобы обеспечить более высокое качество обработки

Специалисты компании Fanhar большое внимание уделяют контролю качества продукции, в частности проводят комплекс испытаний готовых изделий (рис. 6). В процессе производства с помощью автоматического оборудования выполняется двухэтапная проверка: после сборки и после упаковки продукции. В ходе проверки измеряются 


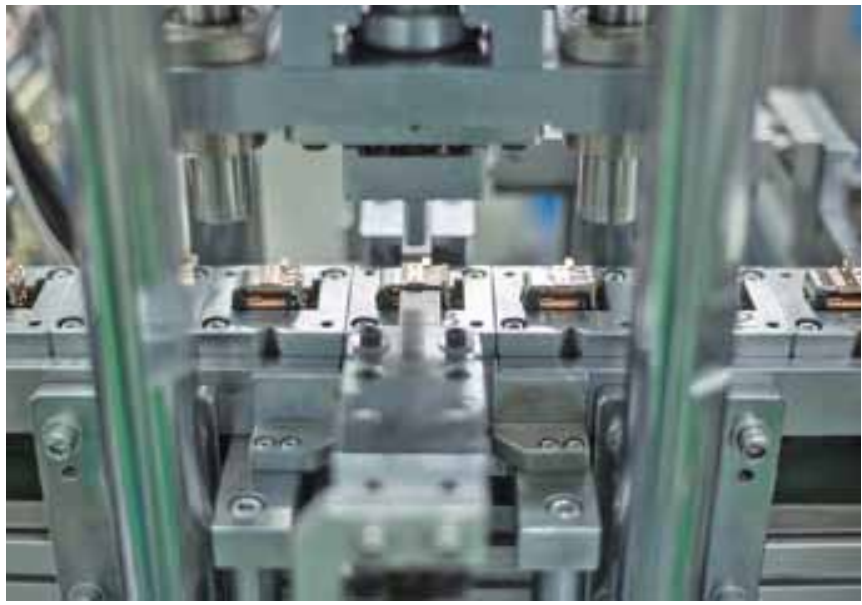

Рис. 5. Автоматическая сборка подвижной пружины

рабочее напряжение реле, время работы, контактное сопротивление, сопротивление катушки, электрическая прочность диэлектрика и другие параметры. Выборочно, по требованию клиента можно проводить испытание на герметичность.

Кроме того, предусмотрены другие виды контроля продукции, в частности испытания на срок службы механической конструкции, электрическую стойкость, перепады

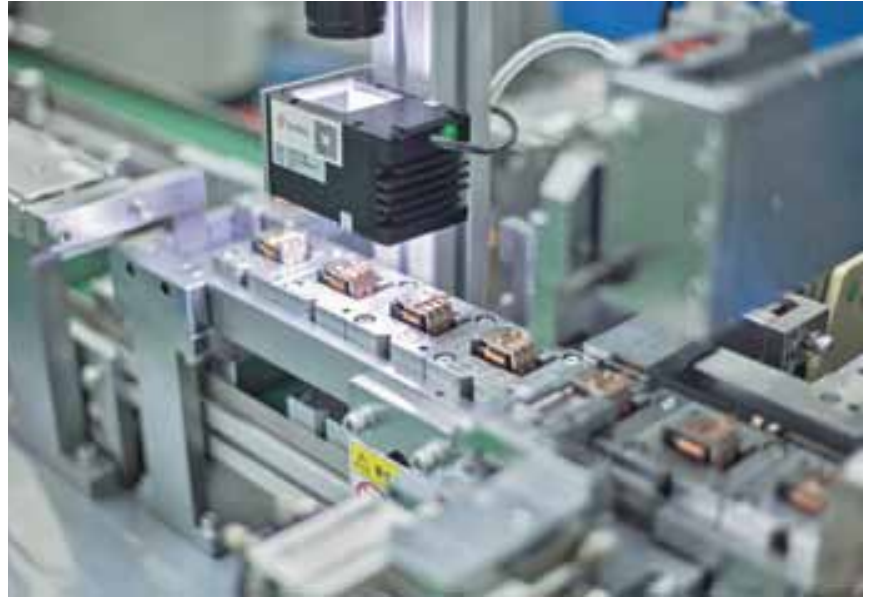

Рис. 6. Автомат визуального обнаружения

температур, устойчивость к падению, ударостойкость и др. С периодичностью раз в три месяца проводятся испытания образцов из партии на долговечность, чтобы проконтролировать стабильность параметров выпускаемой продукции.

Весь ассортимент продукции проходит обязательную сертификацию на требования по безопасности в соответствии со стандартами UL, TUV, CQC и др.

\section{КНИГИ ИЗДАТЕЛЬСТВА «ТЕХНОСФЕРА»}

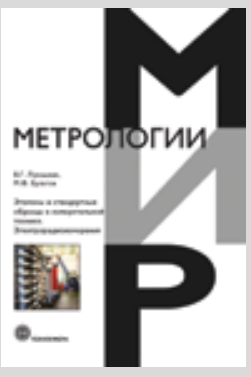

Цена 840 руб.

\section{ЭТАЛОНЫ И СТАНДАРТНЫЕ ОБРАЗЦЫ В ИЗМЕРИТЕЛЬНОЙ ТЕХНИКЕ. ЭЛЕКТРОРАДИОИЗМЕРЕНИЯ}

\author{
Лукашкин В.Г., Булатов М.Ф.
}

Издание осуществлено при финансовой поддержке Федерапьного агентства nо печати и массовым коммуникациям в рамках Федерапьной целевой программы "Купьтура России (2012-2018 годы)"

В книге рассмотрены общие вопросы метрологического обеспечения и единицы физических величин. Изложены основные задачи технических средств метрологического обеспечения в области электрорадиоизмерений. Даны оценки погрешности и неопределенности первичных и рабочих эталонов.

Книга может быть полезна студентам и аспирантам при выборе и обосновании эталонной базы в области электрорадиоизмерений, а также специалистам, занимающимся вопросами разработки, производства и оценки качества средств измерений, контроля и испытаний. 